\title{
PAPEL DE LOS CANNABINOIDES EN EL GLAUCOMA
}

\section{ROLE OF THE CANNABIONOIDS IN GLAUCOMA}

\author{
PINAR-SUEIRO $S^{1}$
}

En las últimas décadas hemos sido testigos de un gran desarrollo en el conocimiento de la patofisiología del glaucoma, de innovadoras técnicas de diagnóstico y detección de progresión precoces. También hemos podido apreciar un avance técnico en el tratamiento quirúrgico y láser de algunos tipos de glaucoma. Sin embargo, en el tratamiento médico seguimos contando con un mismo abanico de familias de fármacos, lo que nos hace responsables de seguir investigando nuevas vías de disminuir la presión intraocular, e incluso ejercer un efecto neuroprotector para garantizar un mejor control de aquellos pacientes que se van mostrando refractarios al efecto de los recursos disponibles para evitar la progresión glaucomatosa.

En la década de los 70 se dispararon los estudios sobre los cannabinoides aplicados al glaucoma, a raíz de observar un claro efecto hipotensor ocular de los mismos (descenso de un 5 a un $45 \%$ de la tensión ocular) en una muestra de voluntarios que ingería o fumaba marihuana (1). El efecto tenía, no obstante, una duración media de 3-4 horas y requería por tanto una frecuente dosificación para mantener tensiones estables. Posteriormente surgieron nuevos estudios con distintas vías de dosificación consiguiendo efectos similares sobre la tensión ocular. Así, se confirmó que el principal ingrediente activo y con mayor efecto psicotrópico de la marihuana (delta-9-tetrahidrocannabinol) disminuía la tensión ocular cuando era administrado de forma intravenosa, oral o inhalado. Respecto a este efecto hipotensor ocular dosis-dependiente, se ha clasificado como respondedores a cannabinoides a un 60$65 \%$ de la población, extrayendo estas conclusiones de una muestra con voluntarios sanos y pacientes con glaucoma (2).

No son escasos, tampoco los estudios que han demostrado un efecto neuroprotector de los canna- binoides. El tetrahidrocannabinol ha demostrado disminuir el efecto neurodegenerativo en modelos de isquemia cerebral en ratas. También se ha demostrado el efecto beneficioso de los cannabinoides al disminuir la degeneración secundaria asociada al glaucoma mediada por la excitotoxicidad del glutamato. Este efecto se ha conseguido reproducir también en modelos experimentales en los que se sometía a las células ganglionares de la retina a stress oxidativo.

Aunque todavía no es bien conocido el mecanismo exacto mediante el cual los cannabinoides pueden actuar sobre la fisiología ocular, en la década de los 90 se describen los dos principales receptores de cannabinoides clonados hasta la fecha, esto es, los rCB1 y rCB2. Los receptores mayoritarios a nivel ocular son los rCB1. Se ha postulado un efecto de los mismos disminuyendo la producción de humor acuoso, y un aumento de la excreción de humor acuoso a través de la malla trabecular y la vía uveoescleral, efecto compatible con el hallazgo de elevadas concentraciones de dichos receptores en el estroma y epitelio no pigmentario del cuerpo ciliar, los vasos sanguíneos del cuerpo ciliar, el músculo ciliar y la malla trabecular. Estos receptores también se han identificado en distintas estructuras de la retina, siendo el marcaje especialmente intenso a nivel de los segmentos externos de los fotorreceptores, y las capas plexiforme interna, externa y nuclear interna, habiéndose asociado a las células ganglionares, amacrinas y horizontales (3).

Se ha propuesto que el efecto neuroprotector se produce fundamentalmente a través de la activación de los receptores tipo CB1. Estos receptores CB1 podrían actuar como neuroprotectores al inhibir los canales de calcio voltaje-dependientes. Sin embargo, no está del todo claro que el efecto neuroprotector aportado por los cannabinoides se encuentre

\footnotetext{
${ }^{1}$ Servicio de oftalmología del Hospital de Cruces. Departamento de Biología Celular. Universidad del País Vasco. Campus de Leioa. Vizcaya. E-mail: luengonosvemos@yahoo.es
} 
exclusivamente a nivel de los receptores CB1, ya que el empleo de CBD, un canabinoide no psicotrópico que no activa los receptores CB1 también demostró un efecto neuroprotector in vivo mediante el bloqueo en la formación de nitrotirosina. Además el cannabidiol no sólo ejerce efectos neuroprotectores per se; también inhibe la degradación del cannabinoide endógeno, araquidonil etanolamida o anandamida (4).

A pesar de estos efectos beneficiosos descritos en el contexto del glaucoma, los cannabinoides no están exentos de efectos indeseados; así, la administración de la mayor parte de los fármacos de la familia de los cannabinoides van asociados a efectos sistémicos adversos, como la hipotensión, taquicardia, palpitaciones y alteraciones psicomotoras. A nivel ocular también se ha asociado a una contracción del músculo ciliar mantenida asociándose a una disminución del rango de acomodación, y opacidades corneales.

Para evitar este tipo de efectos secundarios, se comenzó a ensayar su aplicación tópica. A pesar de la alta liposolubilidad de estos compuestos, lo cual dificultaba tanto su correcta disolución como la absorción, el empleo de ciclodextrinos permitió obviar este problema, con resultados muy esperanzadores. Así, Porcella y cols. (5) demostraron el efecto hipotensor ocular de un agonista sintético de cannabinoides (WIN552122), cuando éste era administrado de forma tópica en pacientes voluntarios refractarios a terapias médicas convencionales.
Antes de desarrollar el estudio, se comprobó en voluntarios sanos la ausencia de efectos secundarios tópicos y sistémicos.

Estos hallazgos sobre el efecto beneficioso de los cannabinoides como hipotensores oculares y por su efecto neuroprotector, nos transmiten un mensaje esperanzador sobre el papel que estos podrían desempeñar en el campo del glaucoma, aunque para poder llegar a confirmar su perfil de seguridad y eficacia serían necesarios ensayos clínicos encaminados a valorar su aplicabilidad en nuestra práctica clínica diaria.

\section{BIBLIOGRAFÍA}

1. Hepler RS, Frank IR. Marihuana smoking and intraocular pressure. JAMA 1971; 217: 1392.

2. Green K. Marijuana smoking vs cannabinoids for glaucoma therapy. Arch Ophthalmol 1998; 116: 1512-1513.

3. Pinar-Sueiro, S, Rodríguez-Puertas, R, Manuel, I, Rivas, A, Vecino, E. Localización de receptores para cannabinoides en el sistema visual como diana terapéutica para la neuropatía óptica glaucomatosa. Soc Esp Neurociencia 2009; S144.

4. Bisogno T, Haus L, De Petrocellis L, Tchilibon S, Ponde $D E$, Brandi I, et al. Molecular targets for cannabidiol and its synthetic analogues: effect on vanilloid VRI receptors and on the cellular uptake and enzymatic hydrolysis of anandamide. Br J Pharmacol 2001; 134: 845-852.

5. Porcella A, Maxia C, Gessa GL, Pani L. The synthetic cannabinoid WIN55212-2 decreases the intraocular pressure in human glaucoma resistant to conventional therapies. Eur J Neurosci 2001; 13: 409-412. 